Pacific Journal of Mathematics

THE STONE-WEIERSTRASS PROPERTY IN BANACH 


\section{THE STONE-WEIERSTRASS PROPERTY IN BANACH ALGEBRAS}

\section{YitZHAK KATZNELSON AND WALTER RUDIN*}

Introduction. Let $A$ be a semi-simple commutative Banach algebra with maximal ideal space $\Delta$. Regarding the elements of $A$ as functions on $A$, we call a subalgebra $B$ of $A$ self-adjoint if corresponding to every $f \in B$ the function $\bar{f}$ defined on $\Delta$ by $\bar{f}(x)=\overline{f(x)}$ is also in $B$; we call $B$ separating if to every pair of distinct points $x_{0}, x_{1} \in \Delta$ there is an $f \in B$ such that $f\left(x_{0}\right)=0, f\left(x_{1}\right)=1$.

If every separating self-adjoint subalgebra of $A$ is dense in $A$, we say that $A$ has the Stone-Weierstrass property.

The Stone-Weierstrass property is related, to some extent at least, to the ideal structure of $A$. For instance, it is obvious that if $A$ has a unit and a closed primary ideal $I$ which is not maximal, then the algebra generated by $I$ and the constants is not dense in $A$. More generally, suppose $A$ is self-adjoint, $I$ is a closed self-adjoint ideal in $A$ which is not the intersection of the regular maximal ideals containing it, and $A / I$ is the direct sum of its radical and a subalgebra $B_{0}$. If $h$ is the canonical homomorphism of $A$ onto $A / I$, then $I+h^{-1}\left(B_{0}\right)$ is a separating self-adjoint subalgebra of $A$ which is not dense in $A$, so that $A$ does not have the $S-W$ property.

Also, it was pointed out by Herz that the Schwartz counterexample [9] to spectral synthesis in $L^{1}\left(R^{3}\right)$ yields immediately an example of a closed, separating, self-adjoint, proper subalgebra of $L^{1}\left(R^{3}\right)$. After Malliavin's solution of the spectral synthesis problem for $L^{1}(\Gamma)$, where $\Gamma$ is any locally compact abelian group, it was natural to investigate the $S-W$ property for these algebras.

In Part I (whose contents were announced in [5]) this is done for $\Gamma=Z$, the additive group of the integers. The general case is settled in Part II; the solution shows that the relation between the $S-W$ property and the ideal structure is, after all, not a very close one. Part III deals with the relation between the self-adjointness of $A$ and the total disconnectedness of $\Delta$.

For convenience of notation, we shall phrase our results on group algebras in $A(G)$ rather than in $L^{1}(\Gamma)$. Here $G$ and $\Gamma$ are dual groups of each other, and $A(G)$ is the algebra of all Fourier transforms of functions in $L^{1}(\Gamma)$. The circle group (the dual of $Z$ ) will be denoted by $T$, so that $A(T)$ is the algebra of all absolutely convergent Fourier series.

Since every locally compact abelian group is locally isomorphic to a

Received April 4, 1960. * Research Fellow of the Alfred P. Sloan Foundation. 
compact group, nothing of interest is lost by restricting our attention to algebras $A(G)$ with $G$ compact.

\section{PART I}

LEMma 1.1. If $g$ and its derivative $g^{\prime}$ are in $A(T)$, and if $\varepsilon>0$, $0<\delta<\pi$, there exists a function $\phi$ on $T$ with the following properties:

(i) $0 \leqq \varphi \leqq 1$;

(ii) $\varphi \equiv 1$ in some neighborhood of $0, \varphi \equiv 0$ outside $(-\delta, \delta)$;

(iii) if $h=\varphi g$, then $|n \hat{h}(n)|<\varepsilon(n=0, \pm 1, \pm 2, \cdots)$.

Here $\hat{h}(n)$ denotes the $n$th Fourier coefficient of $h$ :

$$
\hat{h}(n)=\frac{1}{2 \pi} \int_{-\pi}^{\pi} h(x) e^{-i n x} d x .
$$

Proof. Let $u$ be a continuous odd function on the real line, vanishing outside the segments $(-2,-1)$ and $(1,2)$, such that $\int_{1}^{2} u(s) d s=-1$, and put

$$
\widehat{u}(t)=\frac{1}{2 \pi} \int_{-\infty}^{\infty} u(s) e^{-i s t} d s
$$

Note that

(a) $\hat{u}(0)=0$,

(b) $\hat{u}$ is continuous,

(c) $\|\hat{u}\|_{\infty}<1$, and

(d) $\hat{u}(t) \rightarrow 0$ as $|t| \rightarrow \infty$.

For $r=1,2,3, \cdots$, put $u_{r}(x)=r u(r x)$. Then $\widehat{u}_{r}(t)=\widehat{u}(t / r)$, and the above mentioned properties of $\hat{u}$ show that there exists a sequence of positive integers $r_{i}$ (which must increase sufficiently rapidly), so that

$$
\left|\hat{u}_{r_{1}}(t)+\cdots+\hat{u}_{r_{k}}(t)\right|<1 \quad(k=1,2,3, \cdots ; t \text { real }) .
$$

Take $r_{1}$ and $k$ so large that $r_{1}>2 / \delta$ and

$$
\left(\frac{1}{k}+\frac{2}{\pi r_{1}}\right)\left(\|g\|_{A}+\left\|g^{\prime}\right\|_{A}\right)<\varepsilon .
$$

(The subscripts $A$ indicate that the norms are taken in $A(T)$.) Define

$$
\begin{gathered}
v=\frac{1}{k}\left(u_{r_{1}}+\cdots+u_{r_{k}}\right), \\
\varphi(x)=\int_{-\pi}^{x} v(t) d t .
\end{gathered}
$$

Our construction shows immediately that $\phi$ has properties (i) and (ii) of the lemma. If $h=\phi g$, then $h^{\prime}=v g+\varphi g^{\prime}$. Note that $|\hat{\varphi}(0)| \leqq 2 /\left(\pi r_{1}\right)$ 
and that

$$
|\widehat{\varphi}(n)|=|\hat{v}(n) / n| \leqq|\widehat{v}(n)| \leqq 1 / k
$$

for $n \neq 0$, by (3) and (5). Thus

$$
\begin{aligned}
\sup _{n}|n \hat{h}(n)| & =\left\|\hat{h}^{\prime}\right\|_{\infty} \leqq\|\hat{v}\|_{\infty}\|g\|_{A}+\|\hat{\varphi}\|_{\infty}\left\|g^{\prime}\right\|_{A} \\
& \leqq \frac{1}{k}\|g\|_{A}+\left(\frac{1}{k}+\frac{2}{\pi r_{1}}\right)\left\|g^{\prime}\right\|_{A}<\varepsilon,
\end{aligned}
$$

by (4), and the lemma is proved.

Theorem 1.2. $A(T)$ does not have the Stone-Weierstrass property.

Proof. We shall construct a totally disconnected perfect set $P$ on $T$ and a function $f$, not equivalent to 0 , which vanishes outside $P$, such that $|n \hat{f}(n)|$ is bounded.

Once this is done, we let $B[P]$ be the algebra of all twice continuously differentiable functions $g$ on $T$ such that $g^{\prime}(x)=0$ for every $x \in P$. Since $P$ is totally disconnected, $B[P]$ is a separating (and evidently self-adjoint) subalgebra of $A(T)$. The bounded sequence $\{n \hat{f}(n)\}$ defines a non-zero bounded linear functional $U$ on $L^{1}(Z)$, hence on $A(T)$, and $U$ annihilates $B[P]$ : for $g \in B[P]$, the Fourier series of $g^{\prime}$ converges uniformly, and we have

$$
U g=\sum_{-\infty}^{\infty} \hat{g}(-n) n \hat{f}(n)=\frac{i}{2 \pi} \int_{-\pi}^{\pi} f(x) g^{\prime}(x) d x=0 .
$$

Hence $B[P]$ is not dense in $A(T)$, and the theorem follows.

Now to the construction of $f$ and $P$. Put $f_{0}=1$, and suppose that $f_{i}$ is constructed, so that

$$
\left|n \hat{f}_{i}(n)\right| \leqq 1-2^{-i} \quad(n=0, \pm 1, \pm 2, \cdots) .
$$

Let $I_{i}$ be the largest interval on which $f_{i}$ is identically 1 , let $x_{i}$ be the midpoint of $I_{i}$, and choose $\varphi_{i}$ (by Lemma 1.1) such that

(i) $0 \leqq \varphi_{i} \leqq 1$;

(ii) $\varphi_{i} \equiv 1$ in a neighborhood of $x_{i}, \varphi_{i} \equiv 0$ outside $\left(x_{i}-\delta_{i}, x_{i}+\delta_{i}\right)$, where $\delta_{i}=2^{-i-1}$;

(iii) if $h_{i}=f_{i} \varphi_{i}$, then $\left|n \hat{h}_{i}(n)\right|<2^{-i-1}$ for $n=0, \pm 1, \pm 2, \cdots$.

Define $f_{i+1}=f_{i}\left(1-\varphi_{i}\right)$. Then

$$
\left|n \widehat{f}_{i+1}(n)\right| \leqq\left|n \hat{f}_{i}(n)\right|+\left|n \hat{h}_{i}(n)\right|<1-2^{-i-1},
$$

so that our induction hypothesis (8) is satisfied with $i+1$ in place of $\mathrm{i}$.

The sequence $\left\{f_{i}\right\}$ converges monotonically to a function $f$. Applying the Lebesgue convergence theorem to the computation of $\hat{f}(n),(8)$ shows that $|n \hat{f}(n)| \leqq \mid 1$. Our choice of the points $x_{i}$ shows that $f \equiv 0$ on a 
dense open set $V$. Let $P$ be the complement of $V$. Finally, observe that $f=1$ at those points at which every $\varphi_{i}$ is 0 , and this happens on the complement of a set whose measure does not exceed $2 \sum_{0}^{\infty} \delta_{i}<2 \pi$. Hence $f=1$ on a set of positive measure. This completes the proof.

1.3. It was essential in our preceding construction to have $P$ of positive measure. For suppose $m(P)=0$ and $B[P]$ is defined as in the proof of Theorem 1.2. If $\left\{c_{n}\right\}$ is any bounded sequence such that $\sum_{-\infty}^{\infty} c_{n} \widehat{g}(-n)=0$ for every $g \in B[P]$, and if $f(x) \sim \sum_{n \neq 0}\left(c_{n} / n\right) e^{i n x}$, a computation analogous to (7) shows that $\int f g^{\prime}=0$ for every $g \in B[P]$. It follows that $f$ must vanish outside $P$, and since $m(P)=0, c_{n}=0$ for $n \neq 0$. Then $c_{0}$ much also be 0 , and we conclude that $B[P]$ is dense in $A(T)$.

1.4. However, measure theoretic conditions on $P$ are not enough. To show this, we shall now construct a totally disconnected perfect set $P$ of positive measure, such that $B[P]$ is dense in $A(T)$. Our construction will also show that for every function $g \in A(T)$ there exist differentiable $g_{n}$ such that $\left\|g-g_{n}\right\| \rightarrow 0$ and $g_{n}^{\prime} \rightarrow 0$ a.e.

Put $n_{k}=2^{k+1}, N_{k}=n_{1} n_{2} \cdots n_{k}$, let $k=1,2,3, \cdots$,

$$
L_{k, j}=\left(\frac{2 \pi(j-1)}{N_{k}}, \frac{2 \pi j}{N_{k}}\right),
$$

and let $I_{k}$ be the union of those $L_{k, j}$ which have $j \equiv 1\left(\bmod n_{k}\right)$. The desired set $P$ is the intersection of the complements of the $I_{k}$.

Since $m\left(I_{k}\right)=2 \pi / n_{k}$, we see that $m(P) \geqq \pi$.

Let $g_{k}$ be the characteristic function of $I_{k}$, and define

$$
f_{k}(x)=n_{k} \int_{0}^{x} g_{k}(t) d t .
$$

Then $f_{k}(2 \pi)=2 \pi$, hence $\mathrm{e}^{i f_{k}} \in A(T)$, and since $f_{k}$ is constant on each interval of the complement of $I_{k}, e^{i f_{k}}$ belongs to the closure $\bar{B}$ of $B[P]$.

Next, $f_{k}(x)-x=h_{k}\left(N_{k-1} x\right)$, where $h_{k}(0)=h_{k}(2 \pi)=0, h_{k}$ is linear on $\left[0,2 \pi / n_{k}\right]$ and on $\left[2 \pi / n_{k}, 2 \pi\right], h_{k}$ has period $2 \pi$, and

$$
h_{k}\left(\frac{2 \pi}{n_{k}}\right)=\frac{2 \pi}{N_{k-1}}-\frac{2 \pi}{N_{k}} .
$$

Then $\left\|f_{k}(x)-x\right\|=\left\|h_{k}\left(N_{k-1} x\right)\right\|=\left\|h_{k}\right\|$. Computation of the Fourier coefficients of $h_{k}$ (see 1.5 below) shows that $\left\|h_{k}\right\| \rightarrow 0$ as $k \rightarrow \infty$. It follows that

$$
\left\|e^{i x}-e^{i f_{k}(x)}\right\|=\left\|1-e^{i\left(f_{k}(x)-x\right)}\right\| \rightarrow 0
$$

on $k \rightarrow \infty$, so that $\bar{B}$ contains the function $e^{i x}$. 
Similarly, $e^{-i x} \in \bar{B}$, hence $\bar{B}=A(T)$, and $B[P]$ is dense in $A(T)$.

1.5. Suppose $0<a<1 / 2, b>0$. Let $h$ be linear on $[0, a]$ and on $[a, 2 \pi]$, such that $h(0)=h(2 \pi)=0$, and $h(a)=b$. Then

$$
h(x)=\frac{b}{2}-\frac{b}{2 \pi-a} \sum_{n \neq 0} \frac{1-e^{-i n a}}{n^{2} a} e^{i n x} .
$$

Hence $\|h\|$ (the norm being taken in $A(T)$ ) is bounded by $C b \log 1 / a$, where $C$ is an absolute constant.

1.6. In our proof of Theorem 1.2 we could have chosen the intervals on which $\varphi_{i} \equiv 1$ so that the resulting set $P$ satisfies a certain arithmetic condition which assures that $P$ is a set of spectral synthesis. The condition we have in mind is due Herz [3; Theorem 6.5]: there should be an increasing sequence of integers $n_{k}$ such that a point $2 \pi j / n_{k}$ either lies in $P$ or its distance from $P$ is at least $2 \pi / n_{k}$.

If $P$ is so constructed, let $I$ be the ideal in $A(T)$ of all functions vanishing on $P$. Since $P$ is a set of spectral synthesis, $I$ lies in the closure $\bar{B}$ of $B[P], \bar{B} / I$ is a proper subalgebra of $A(T) / I$, and the latter algebra is semi-simple. Also, $A(T)$ has no closed primary ideals. We conclude:

$A(T) / I$ is a semi-simple Banach algebra, without closed primary ideals, which is not spanned by its set of idempotents, although its maximal ideal space, $P$, is totally disconnected.

\section{PART II}

THEOREM 2.1. If $A$ is a semi-simple commutative Banach algebra which is spanned by its set of idempotents, then $A$ has the StoneWeierstrass property.

Proof. Let $B$ be the closure of a separating self-adjoint subalgebra $B_{0}$ of $A$. (Note that we do not assume that $B$ is self-adjoint; see 3.3.) Let $\Delta(A)$ and $\Delta(B)$ be the maximal ideal spaces of $A$ and $B$. Since $B$ is separating, $\Delta(A) \subset \Delta(B)$.

For any $f \in B$, the norm of $f$ is the same whether we consider $f$ as an element of $A$ or of $B$. Hence the two spectral norms of $f$ (in $A$ and in $B$ ) are the same, so that

$$
\sup _{x \in \Delta(B)}|f(x)|=\sup _{x \in \Delta(A)}|f(x)|(f \in B) .
$$

In other words, the Silov boundary $S$ of $B$ [6; p. 80] lies in $\Delta(A)$.

The equation $\left|e^{f}\right|=e^{R e f}$ shows that (9) holds with the real or imaginary part of $f$ in place of $|f|$. Since $B_{0}$ is self-adjoint on $\Delta(A)$, this 
maximum modulus property shows that $B_{0}$ is also self-adjoint as an algebra of functions on $\Delta(B)$. Since $B_{0}$ is dense in $B, B_{0}$ separates points on $A(B)$, and the Stone-Weierstrass theorem implies that every continuous function on $\Delta(B)$ can be uniformly approximated by elements of $B_{0}$. Thus $S=\Delta(B)$, and we conclude: $\Delta(B)=\Delta(A)$.

Since $A$ is spanned by its idempotents, $\Delta(A)$ is totally disconnected. Silov's theorem on idempotents [10] thus applies to $B$ and shows that $B$ contains every idempotent of $A$. Hence $B=A$, and the theorem is proved.

TheOREm 2.2. Let $G$ be a compact abelian group. Then $A(G)$ has the Stone-Weierstrass property if and only if $G$ is totally disconnected.

Proof. One half of the theorem is an immediate corollary of Theorem 2.1.

To prove the other half, suppose $G$ is not totally disconnected. Its dual group $\Gamma$ then contains an infinite cyclic group $\Lambda$ which can be mapped isomorphically onto $Z$. Regarding $Z$ as a subgroup of $R_{a}$ (the additive group of the real numbers, with the discrete topology), the divisibility of $R_{d}$ [4] implies that our isomorphism of $A$ onto $Z$ can be extended to a homomorphism of $\Gamma$ into $R_{d}$. The duality theory for compact and discrete abelian groups now shows that $G$ contains a compact subgroup $K$ whose dual group $\hat{K}$ is a subgroup of $R_{a}$, and that therefore $K$ is a homomorphic image of the Bohr compactification of $R$. It follows that $K$ contains a dense one-parameter subgroup $J$.

We now use Theorem 1.2 to prove that $A(K)$ does not have the $S-W$ property.

Note that a continuous function $f$ on $K$ belongs to $A(K)$ if and only if its restriction to $J$ is of the form

$$
f(\varphi(t))=\sum_{s \in \hat{K}} a(s) e^{i s t}
$$

with $\sum|a(s)|<\infty$; here $\varphi$ is a fixed continuous isomorphism of $R$ onto $J$. Conversely, every function of the form (10) has a continuous extension to $K$.

A lemma of Wiener $[11 ;$ p. 80] implies that the functions $f(\varphi)$ of the form (10) are locally the same as the members of $A(T)$. Let $\alpha=$ $[-\pi+\varepsilon, \pi-\varepsilon]$, for some fixed $\varepsilon>0$. Choose $P \subset \alpha$, as in the proof of Theorem 1.2, so that $B[P]$ is not dense in $A(T)$, and let $B_{1}$ be the algebra of all $f \in A(K)$ such that $f(\varphi(t))$ coincides with a function in $B[P]$ on $\alpha$. Then $B_{1}$ is a separating self-adjoint subalgebra of $A(K)$ which is not dense in $A(K)$, and $A(K)$ does not have the $S-W$ property.

Finally, we take all $f \in A(G)$ whose restriction to $K$ lies in $B_{1}$, and 
we obtain a separating self-adjoint subalgebra of $A(G)$ which is not dense in $A(G)$.

This completes the proof.

2.3. Suppose $G$ is a totally disconnected infinite compact abelian group, $E$ is a compact subset of $G$ which is not of spectral synthesis (such sets exist [7]), $I$ is the ideal in $A(G)$ consisting of all $f$ which vanish on $E$, and $I_{0}$ is the closure of the ideal consisting of all $f$ which vanish on a neighborhood of $E$.

Define $B=A(G) / I_{0}$. If the idempotents in $B$ spanned a proper closed subalgebra $B^{\prime}$ of $B$, the inverse image of $B^{\prime}$ under the canonical homomorphism of $A(G)$ onto $B$ would be a proper, closed, separating, self-adjoint subalgebra of $A(G)$, in contradiction to Theorem 2.2. The radical of $B$ is $I / I_{0}$, which by a theorem of Helson [2], is infinite dimensional. We conclude:

$B$ is a commutative Banach algebra which is spanned by its idempotents and whose radical $R$ is infinite dimensional.

We shall show, furthermore, that $B$ has no subalgebra $C$, algebraically isomorphic to $B / R$, such that $B$ is the direct sum $C+R$.

Thus the Wedderburn principal theorem does not hold for $B$.

Feldman has constructed an algebra, spanned by its idempotents, with one dimensional radical $R$, which is not the direct sum of $R$ and any closed subalgebra; however, his algebra is the direct sum of $R$ and a non-closed subalgebra [1; Theorem 6.1].

Suppose $C$ is a subalgebra of $B$, and $B=C+R$. Let $h$ be the natural isomorphism of the semi-simple Banach algebra $B / R$ onto $C$ (i.e., into $B$ ). Let $e^{\prime}, e^{\prime \prime}$ be the characteristic functions of disjoint compact sets $E^{\prime}, E^{\prime \prime}$ whose union is $E$, and define $B^{\prime}=e^{\prime} B, C^{\prime}=e^{\prime} C, R^{\prime}=e^{\prime} R$. A result of Bade and Curtis [1; Theorems 3.7, 3.9], combined with the fact that $A(G)$ has no primary ideals, shows that $E^{\prime}$ can be chosen so that

$(\alpha)$ the restriction of $h$ to $e^{\prime} \cdot(B / R)=B^{\prime} / R^{\prime}$ is continuous,

( $\beta) \quad B^{\prime}$ is not semi-simple.

Since $h^{-1}$ is continuous (by the definition of the quotient norm), $(\alpha)$ implies that $C^{\prime}=h\left(B^{\prime} / R^{\prime}\right)$ is a closed subalgebra of $B^{\prime}$. Since

(a) $C^{\prime}$ contains all idempotents of $B^{\prime}$,

(b) these idempotents span $B^{\prime}$ (by the same reasoning that applied to $B$ ), and

(c) $C^{\prime}$ is closed, we conclude that $C^{\prime}=B^{\prime}$, so that $B^{\prime}$ is semisimple, in contradiction to $(\beta)$.

The preceding argument yields a more general result:

THEOREM 2.4. Suppose $A$ is a regular commutative Banach algebra without primary ideals, and suppose $E$ is a compact subset of the 
maximal ideal space of $A$. If $B=A / I_{0}(E)$ is spanned by its idempotents and if $B$ is not semi-simple, then the Wedderburn theorem does not hold for $B$.

Bade and Curtis, in an as yet unpublished paper "The Wedderburn decomposition of commutative Banach algebras", have constructed other examples of commutative Banach algebras in which the radical cannot. be split off algebraically.

\section{PART III}

3.1. The standard examples of non-self-adjoint Banach algebras: involve analytic functions of one or more complex variables, and their maximal ideal spaces are at least two-dimensional. Before turning to the construction of examples with totally disconnected maximal ideal space, we insert two remarks.

(a) If $A$ is semi-simple and self-adjoint, then there is a constant $M$ such that $\|\bar{f}\| \leqq M\|f\|$ for every $f \in A$.

Indeed, considering $A$ as a Banach space over the real field, the map $f \rightarrow \bar{f}$ is linear; the closed graph theorem applies (since $A$ is semisimple) and shows that this map is continuous.

(b) If a semi-simple Banach algebra $A$ has a dense self-adjoint subalgebra $B$, and if the map $f \rightarrow \bar{f}$ is bounded on $B$, then $A$ is selfadjoint.

This is obvious. (It was tacitly used in 2.3, in the assertion that $B^{\prime}$ is self-adjoint.) We mention (b) mainly because of the two examples which follow. In 3.2 we construct an algebra which is not self-adjoint, although the map $f \rightarrow \bar{f}$ is bounded on a separating subalgebra; the algebra constructed in 3.3 contains a dense self-adjoint subalgebra although it is not itself self-adjoint. In both examples, the maximal ideal spaces are totally disconnected.

3.2. Let $\left\{z_{n}\right\}$ be a sequence of complex numbers, with $0<\left|z_{n}\right|<1$, such that $z_{n} \rightarrow 0$ as $n \rightarrow \infty$, and such that the sequence $\left\{z_{n}|| z_{n} \mid\right\}$ is dense on the unit circle.

Let $A$ be the algebra of all sequences $a=\left\{a_{n}\right\}, n=1,2,3, \cdots$, with. termwise addition and multiplication, for which the limit

$$
L(a)=\lim _{n \rightarrow \infty} \frac{a_{n}}{z_{n}}
$$

exists as a finite number; norm $A$ by

$$
\|a\|=\sup _{n}\left|\frac{a_{n}}{z_{n}}\right|
$$

Then $A$ is a Banach algebra. Let $B$ be the set of all $a \in A$ for which 
$L(a)=0$. Since $L(a b)=0$ whenever $a \in A$ and $b \in B, B$ is a closed ideal in $A$. Furthermore, if $e_{k} \in A$ is the sequence whose $K^{t h}$ term is 1 , while all others are 0 , it is easily verified that $B$ is the closure of the set of all finite linear combinations of the $e_{k}$; in other words, $B$ is spanned by the idempotents of $A$.

Let $h$ be a homomorphism of $A$ onto the complex field. If $h(B)=0$, then $[h(a)]^{2}=h\left(a^{2}\right)=0$ for every $a \in A$ (since $a^{2} \in B$ ), a contradiction. Thus $h\left(e_{k}\right) \neq 0$ for some $k$, and $h\left(e_{k}\right) h(a)=h\left(e_{k} a\right)=a_{k} h\left(e_{k}\right)$, so that $h(a)=$ $a_{k}$ for all $a \in A$.

It follows that $A$ is semi-simple, and that its maximal ideal space is discrete and countable. By 3.1 (b), $B$ is self-adjoint.

Suppose next that $a \in A$ and $a$ is real. Then

$$
L(\alpha)=\lim _{n \rightarrow \infty} \frac{a_{n}}{\left|z_{n}\right|} \cdot \frac{\left|z_{n}\right|}{z_{n}} .
$$

Since $\left\{\left|z_{n}\right| / z_{n}\right\}$ is dense on the unit circle, and since $a_{n}$ is real, this can exist only when it is 0 . Hence $a \in B$.

Thus if $a \in A$ and $\bar{a} \in A$, then $a+\bar{a} \in B$ and $i(a-\bar{a}) \in B$, so that $a \in B$. We summarize:

$A$ is a commutative semi-simple Banach algebra whose maximal ideal space is discrete and countable; $A$ contains a proper, closed, separating ideal $B$ which consists precisely of the self-adjoint elements of $A$.

The non-self-adjoint algebra $A$ is, in turn, a closed ideal in the self-adjoint algebra $A_{1}$ which consists of all sequences a such that $\|a\|=\sup _{n}\left|a_{n}\right| z_{n} \mid<\infty$.

The last assertion follows from the inclusions $A \cdot A_{1} \subset B \subset A$.

3.3. Our next example is a regular semi-simple commutative Banach algebra $A$ which is not self-adjoint, although it is spanned by its idempotents.*

Since the algebra of all finite linear combinations of idempotents is always self-adjoint, we see that $A$ contains a dense self-adjoint subalgebra.

Define $w_{n}=1$ if $n \leqq 0, w_{n}=1+\log (n+1)$ if $n \geqq 1$, and let $A_{0}$ be the algebra of all functions $f$ of the form

$$
f(x)=\sum_{-\infty}^{\infty} a_{n} e^{i n x}
$$

for which the norm

$$
\|f\|_{0}=\sum_{-\infty}^{\infty}\left|a_{n}\right| w_{n}
$$

is finite.

* Added in proof. Other algebras with this property were found by Coddington (Proc. Amer. Math. Soc. 8 (1957), 258-261). 
The inequality $w_{n+m} \leqq w_{n} w_{m}$ shows that $A_{0}$ is a Banach algebra and it is easily verified that its maximal ideal space is unit circle $T$. Since $A_{0}$ contains every $f$ with two continuous derivatives, $A_{0}$ is regular. i.e., given any two disjoint compact sets $C_{0}$ and $C_{1}$ on $T$, there exists $f \in A_{0}$ such that $f=0$ on $C_{0}, f=1$ on $C_{1}$. Wermer has pointed out that algebras like $A_{0}$ furnish simple examples of non-self-adjoint algebras with one-dimensional maximal ideal space.

Given a rapidly increasing sequence of positive integers $p_{k}(k=$ $1,2,3, \cdots)$, let

$$
L_{j, k}^{-}=\left(\frac{2 \pi(j-1)}{p_{k}^{2}}, \quad \frac{2 \pi j}{p_{k}^{2}}\right) \quad\left(1 \leqq j \leqq p_{k}^{2}\right),
$$

let $I_{k}$ be the union of those $L_{j, k}$ with $j \equiv 1\left(\bmod p_{k}\right)$, and define $P$ to. be the intersection of the complements of the sets $I_{k}$.

Our desired algebra $A$ is the restriction of $A_{0}$ to $P$.

We first prove that $A$ is spanned by its idempotents. As in 1.4, let $g_{k}$ be the characteristic function of $I_{k}$, and put $f_{k}(t)=p_{k} \int_{0}^{x} g_{k}(t) d t$. Then $e^{i f_{k}} \in A_{0}$, and $f_{k}(x)-x=h_{k}\left(p_{k} x\right)$, where $h_{k}(0)=h_{k}(2 \pi) \stackrel{J_{0}}{=} 0, h_{k}$ is linear on $\left[0,2 \pi / p_{k}\right]$ and on $\left[2 \pi / p_{k}, 2 \pi\right]$, and

$$
h_{k}\left(\frac{2 \pi}{p_{k}}\right)=\frac{2 \pi}{p_{k}}-\frac{2 \pi}{p_{k}^{2}} .
$$

We have

$$
\left\|h_{k}\left(p_{k} x\right)\right\|_{0}=\sum_{-\infty}^{\infty}\left|\hat{h}_{k}(n)\right| w_{n p_{k}} .
$$

The Fourier series of $h_{k}$ is exhibited in 1.5 , with $a=2 \pi / p_{k}, b=$ $2 \pi\left(p_{k}-1\right) / p_{k}^{2}$, and a simple computation now shows that

$$
\left\|h_{k}\left(p_{k} x\right)\right\|_{0}<C \frac{\log ^{2} p_{k}}{p_{k}}
$$

which tends to 0 as $k \rightarrow \infty$. As in 1.4 , it follows that $\left\|e^{i x}-e^{i f_{k}(x)}\right\|_{0} \rightarrow 0$ as $k \rightarrow \infty$.

Since $e^{i f_{k}}$ is constant on each arc contiguous to $I_{k}$, the restriction of $e^{i f_{k}}$ to $P$ is a finite linear combination of idempotents of $A$. It follows that the restriction of $e^{i x}$ to $P$ is in the span of the idempotents; the same is true of $e^{-i x}$, and hence $A$ is spanned by its idempotents.

It remains to be shown that $\left\{p_{k}\right\}$ can be so chosen that $A$ will not be self-adjoint. We do this inductively.

Let $P_{k}$ be the complement of $I_{1} \cup \cdots \cup I_{k}$, and let $A_{k}$ be the restriction of $A_{0}$ to $P_{k}$. We claim that $A_{k}$ is not self-adjoint. To prove this, note that $A_{k}$ contains the restriction to $P_{k}$ of all $f(x)=\sum_{-\infty}^{0} a_{n} e^{i n x}$ with $\sum_{-\infty}^{0}\left|a_{n}\right|<\infty$. If $A_{k}$ were self-adjoint, it would follow that $A_{k}$ consists of all restrictions of functions in $A(T)$ to $P_{k}$, and since $P_{k}$ 
contains an arc, this would imply that $A(T)$ and $A_{0}$ coincide locally. Being regular, these algebras would therefore have to be the same, a contradiction.

Let $c(n, k)$ be the $A_{k}$-norm of the restriction of $e^{i n x}$ to $P_{k}$, i.e.,

$$
\begin{aligned}
& c(n, k)=\inf \left\{\|f\|_{0}: f(x)=e^{i n x} \text { on } P_{k}\right\} \\
& \qquad(n=0, \pm 1, \pm 2, \cdots) .
\end{aligned}
$$

Since $c(n, k)=1$ for $n \leqq 0$ and $A_{k}$ is not self-adjoint, 3.1 (b) shows that $c(n, k)$ is unbounded as $n \rightarrow+\infty$. In particular, there exists $n_{k}$ such that

$$
c\left(n_{k}, k\right)>k \text {. }
$$

We now claim that there exists $\delta_{k}>0$ with the following property: If $0 \leqq n \leqq k$, if $V$ is an open set with $m(V)<\delta_{k}$, if $g \in A_{0}$ and $g(x)=e^{i n x}$ on $P_{k}-V$, then $\|g\|_{0}>c(n, k)-2^{-k}$.

Suppose this is false. Then there exists

(i) an integer $n, 0 \leqq n \leqq n_{k}$,

(ii) open sets $V_{r}$ with $m\left(V_{r}\right)<2^{-r}(r=1,2,3, \cdots)$,

(iii) $g_{r} \in A_{0}$ satisfying $g_{r}(x)=e^{i n x}$ on $P_{k}-V_{r}$, such that

(iv) $\left\|g_{r}\right\|_{0} \leqq c(n, k)-2^{-k}$.

By (iv), the diagonal process yields a sequence $\left\{r_{i}\right\}$ such that $\hat{g}_{r_{i}}(m)$ converges, say to $a_{m}$, for $m=0, \pm 1, \pm 2, \cdots$. Put

$$
g(x)=\sum_{-\infty}^{\infty} a_{m} e^{i m x}
$$

Then $g \in A_{0}$, and

$$
\|g\|_{0} \leqq c(n, k)-2^{-k}
$$

For every $f \in L(T), \int g_{r_{i}} f \rightarrow \int g f$ as $i \rightarrow \infty$. Combined with (ii) and (iii), this shows that $g(x)=e^{i n x}$ a.e. on $P_{k}$, and since $g$ is continuous, this equality holds everywhere on $P_{k}$. But then (14) contradicts (11), the definition of $c(n, k)$.

Having determined $\delta_{k}$, we choose $P_{k+1}$ so that $2 \pi-m\left(P_{k+1}\right)<(1 / 2) \delta_{k}$, i.e., so that $m\left(I_{k+1}\right)<(1 / 2) \delta_{k}$, and we furthermore subject the sequence $\left\{P_{k}\right\}$ to the requirement

$$
m\left(I_{k+1}\right)+m\left(I_{k+2}\right)+\cdots<\delta_{k} .
$$

Then $m\left(P_{k}-P\right)<\delta_{k}$ for every $k$, and it follows that the $A$-norm of the restriction of $e^{i n_{k} x}$ to $P$ is not less than $c\left(n_{k}, k\right)-1$, i.e., not less than $k-1$, by (12). Since the restrictions of the trigonometric polynomials to $P$ are dense in $A, 3.1$ (b) implies that $A$ is not selfadjoint. This completes the proof. 
3.4. We conclude with a theorem which shows that under certain conditions the hypothesis of self-adjointness can be dropped from the Stone-Weierstrass theorem (a special case of this appeared in [8]):

THEOREM. If $A$ is a semi-simple commutative Banach algebra which is spanned by its idempotents and whose maximal ideal space contains no perfect subset, then every separating subalgebra of $A$ is dense in $A$.

Proof. Let $B$ be a closed separating subalgebra of $A$, and denote the maximal ideal spaces of $A$ and $B$ by $\Delta(A)$ and $\Delta(B)$.

Fix $x_{0}, x_{1} \in \Delta(A), x_{0} \neq x_{1}$. There exists $f \in B$ such that $f\left(x_{0}\right)=0$, $f\left(x_{1}\right)=1$. Since $\Delta(A)$ has no perfect subsets, $f(\Delta(A)) \cup\{0\}$ is a compact countable subset of the complex plane [8].

Suppose there is a point $y \in \Delta(B)$ such that $f(y) \neq 0$ and $f(y)$ is not in $f(\Delta(A))$. Then there is a polynomial $P(z)=\sum_{1}^{N} a_{n} z^{n}$ such that

$$
|P(f(y))|>\sup \{|f(z)|: z \in f(\Delta(A))\},
$$

and the function $P(f)$, which belongs to $B$, does not attain its maximum modulus (relative to $\Delta(B)$ ) on $\Delta(A)$. But the Silov boundary of $B$ is in $\Delta(A)$, as in the proof of Theorem 2.1, and we have a contradiction.

Thus $f(\Delta(B))=f(\Delta(A))$.

We can therefore find disjoint open sets $V, W$ in the plane, such that $0 \in V, 1 \in W, f(\Delta(B)) \subset V \cup W$. Define $g=0$ on $V, g=1$ on $W$. The theorem on analytic functions in Banach algebras $[6 ; p .78]$ shows that $g(f) \in B$. That is to say, $B$ contains the characteristic function of a compact open set $E \subset \Delta(A)$ such that $x_{1} \in E$ but $x_{0} \notin E$.

Since $x_{0}, x_{1}$ were arbitrary, the sets $E$ so obtained form a basis for the topology of $\Delta(A)$. This implies that $B$ contains the characteristic function of every compact open set in $\Delta(A)$, and so $B=A$.

This proof, unlike our proof of Theorem 2.1, does not use Silov's theorem on idempotents. In fact, the preceding proof establishes Silov's theorem in the special case in which $\Delta(A)$ contains no perfect set.

\section{REFERENCES}

1. W. G. Bade and P.C. Curtis, Jr., Homomorphisms of commutative Banach algebras, Amer. Math. J., 82 (1960), 589-608.

2. Henry Helson, On the ideal structure of group algebras, Ark. Mat. 2 (1952), 83-86.

3. C. S. Herz, The spectral theory of bounded functions, Trans. Amer. Math. Soc., 94 (1960), 181-232.

4. Irving Kaplansky, In finite Abelian groups, Ann Arbor, 1954.

5. Yitzhak Katznelson, Some examples in the theory of absolutely convergent Fourier series, Abstract 564-148, Notices of Amer. Math. Soc., Dec. 1959. 
6. L. H. Loomis, An Introduction to Abstract Harmonic Analysis, New York, 1953.

7. Paul Malliavin, Sur l'impossibilité de la synthèse spectrale sur les groupes abéliens non compacts, Publications Mathématiques de 1'Institut des Hautes Etudes Scientifiques Paris, (1959), 61-68.

8. Walter Rudin, Continuous functions on compact spaces without perfect subsets, Proc. Amer. Math. Soc. 8 (1957), 39-42.

9. Laurent Schwartz, Sur une propriété de synthèse spectrale dans les groupes non com. pacts, C. R. Acad. Sci. Paris 227 (1948), 424-426.

10. G. E. Silov, On decomposition of a commutative normed ring in a direct sum of ideals, Mat. Sbornik N. S. 32 (74) (1953), 353-364.

11. Norbert Wiener, The Fourier Integral and Certain of its Applications, Cambridge University Press, 1933.

UNIVERSITY OF CALIFORNIA

AND

UNIVERSITY OF WISCONSIN 



\section{PACIFIC JOURNAL OF MATHEMATICS}

\section{EDITORS}

Ralph S. Phillips

Stanford University

Stanford, California

F. H. BrownelL

University of Washington

Seattle 5 , Washington
A. L. Whiteman

University of Southern California Los Angeles 7. California

L. J. PAIGE

University of California

Los Angeles 24, California

ASSOCIATE EDITORS
E. F. BECKENBACH
D. DERRY
H. L. ROYDEN
E. G. STRAUS
T. M. CHERRY
M. OHTSUKA
E. SPANIER
F. WOLF

\section{SUPPORTING INSTITUTIONS}

UNIVERSITY OF BRITISH COLUMBIA

CALIFORNIA INSTITUTE OF TECHNOLOGY

UNIVERSITY OF CALIFORNIA

MONTANA STATE UNIVERSITY

UNIVERSITY OF NEVADA

NEW MEXICO STATE UNIVERSITY

OREGON STATE COLLEGE

UNIVERSITY OF OREGON

OSAKA UNIVERSITY

UNIVERSITY OF SOUTHERN CALIFORNIA
STANFORD UNIVERSITY

UNIVERSITY OF TOKYO

UNIVERSITY OF UTAH

WASHINGTON STATE COLLEGE,

UNIVERSITY OF WASHINGTON

AMERICAN MATHEMATICAL SOCIETY

CALIFORNIA RESEARCH CORPORATION

HUGHES AIRCRAFT COMPANY

SPACE TECHNOLOGY LABORATORIES

NAVAL ORDNANCE TEST STATION

Printed in Japan by International Academic Printing Co., Ltd., Tokyo, Japan

Reprinted 1966 in the United States of America 


\section{Pacific Journal of Mathematics}

\section{Vol. 11, No. $1 \quad$ November, 1961}

A. A. Albert, Generalized twisted fields ............................ 1

Richard Arens, Operational calculus of linear relations ................... 9

John Herbert Barrett, Disconjugacy of a self-adjoint differential equation of the fourth order ....................................... 25

Paul Richard Beesack, Hardy's inequality and its extensions ............... 39

Julius Rubin Blum and David Lee Hanson, On invariant probability measures.

II .............................................

Robert Allen Bonic, Symmetry in group algebras of discrete groups.......... 73

R. Creighton Buck, Multiplication operators ...................... 95

Jack Gary Ceder, Some generalizations of metric spaces ................. 105

Meyer Dwass, Random crossings of cumulative distribution functions ......... 127

Albert Edrei, Wolfgang H. J. Fuchs and Simon Hellerstein, Radial distribution and

deficiencies of the values of a meromorphic function ............... 135

William Cassidy Fox, Harmonic functions with arbitrary local singularities ..... 153

Theodore Thomas Frankel, Manifolds with positive curvature ............... 165

Avner Friedman, A strong maximum principle for weakly subparabolic

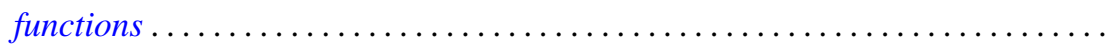

Watson Bryan Fulks and J. O. Sather, Asymptotics. II. Laplace's method for

multiple integrals ......................................

Adriano Mario Garsia and Eugene Richard Rodemich, An embedding of Riemann

surfaces of genus one ..................................... 193

Irving Leonard Glicksberg, Weak compactness and separate continuity......... 205

Branko Grünbaum, On a conjecture of H. Hadwiger .................. 215

Frank J. Hahn, On the action of a locally compact group on $E_{n} \ldots \ldots \ldots \ldots \ldots . . \ldots 221$

Magnus R. Hestenes, Relative hermitian matrices ..................... 225

G. K. Kalisch, On similarity invariants of certain operators in $L_{p} \ldots \ldots \ldots \ldots .247$

Yitzhak Katznelson and Walter Rudin, The Stone-Weierstrass property in Banach

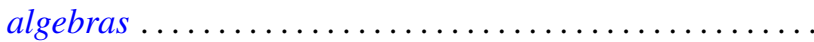

Samir A. Khabbaz, The subgroups of a divisible group $G$ which can be represented as intersections of divisible subgroups of $G \ldots \ldots \ldots \ldots \ldots \ldots \ldots \ldots \ldots . \ldots \ldots 7$

Marvin Isadore Knopp, Construction of a class of modular functions and forms .......................................... 275

Charles Alan McCarthy, Commuting Boolean algebras of projections .......... 295

T. M. MacRobert, Transformations of series of E-functions ................ 309

Heinz Renggli, An inequality for logarithmic capacities ................. 313

M. S. Robertson, Applications of the subordination principle to univalent functions .......................................... 315

David Sachs, Partition and modulated lattices ..................... 325

Frank S. Scalora, Abstract martingale convergence theorems ............... 347

Elbert A. Walker, Torsion endomorphic images of mixed Abelian groups ........ 375

Morgan Ward, The prime divisors of Fibonacci numbers................. 379

Charles R. B. Wright, On the nilpotency class of a group of exponent four....... 387 